\title{
BMJ Open Utilisation of district health information system and its associated factors among health professionals working at public health facilities of the southwest of Ethiopia: cross-sectional survey
}

\author{
Shuma Gosha Kanfe (D) , ${ }^{1}$ Gebiso Roba Debele, ${ }^{2}$ Robera Demissie Berhanu, ${ }^{3}$ \\ Habtamu Setegn Ngusie (D) , ${ }^{1}$ Mohammedjud Hassen Ahmed ${ }^{1}$
}

To cite: Kanfe SG, Debele GR, Berhanu RD, et al. Utilisation of district health information system and its associated factors among health professionals working at public health facilities of the southwest of Ethiopia: crosssectional survey. BMJ Open 2021;11:e046578. doi:10.1136/ bmjopen-2020-046578

- Prepublication history and additional supplemental material for this paper are available online. To view these files, please visit the journal online (http://dx.doi.org/10.1136/ bmjopen-2020-046578).

Received 05 November 2020 Accepted 29 July 2021

Check for updates

(C) Author(s) (or their employer(s)) 2021. Re-use permitted under CC BY-NC. No commercial re-use. See rights and permissions. Published by BMJ.

${ }^{1}$ Health Informatics, Mettu University, Mettu, Ethiopia

${ }^{2}$ Public Health, Mettu University, Mettu, Ethiopia

${ }^{3}$ Clinical Nursing, Mettu University, Mettu, Ethiopia

Correspondence to

Shuma Gosha Kanfe; shumagosha33@gmail.com

\section{ABSTRACT}

Objectives To assess utilisation of district health information system and its associated factors among health professionals in the southwest of Ethiopia, 2020.

Setting Public health facilities in the southwest of Ethiopia.

Participants A facility-based cross-sectional study was conducted among a sample of 260 participants.

Main outcome measures The main outcome measure was utilisation of the district health information system. Results Overall, 149 (57.3\%) of study participants had good utilisation of district health information systems (95\% Cl 50 to 64.2). Sufficient skills (Adjusted Odds Ratio (AOR) 3.83, 95\% Cl 1.92 to 7.64), being trained (AOR 3.90, $95 \% \mathrm{Cl} 1.95$ to 7.79 ), high motivation (AOR $3.93,95 \% \mathrm{Cl}$ 1.99 to 7.76 ), feedback provided (AOR $2.93,95 \% \mathrm{Cl} 1.53$ to 5.77 ) and regular supervision (AOR 3.06, 95\% Cl 1.56 to 6.01 ) were associated with utilisation of district health information systems.

Conclusions In general, more than half of the respondents had good utilisation of district health information systems. Providing regular supportive supervision and feedback, having good skills on district health information system use, high motivation and being trained on district health information system will help to bring good utilisation of district health information system for decision making.

\section{BACKGROUND}

World Health Organization (WHO) views district health information systems (DHIS) as one of the building blocks of the health system. ${ }^{12}$ DHIS, through the use of DHIS software tools, is used to aggregate statistical data collection, validation, analysis, management and presentation providing data analytics and management platform. ${ }^{34}$ This is due to the use of electronic forms for data collection, which provides more efficient and accurate collection of data at the national level with better quality control measures. ${ }^{35}$ The use of DHIS as health information is expected
Strengths and limitations of this study

- This is the first study in Ethiopia assessing the utilisation of the district health information system for decision making among health professionals.

- The study was conducted in wide study areas including different health facilities such as referral hospitals, primary hospitals and health centres. This strengthens the generalisability of the findings.

- It is difficult to know about the precedence of the problem in detail because the study was cross-sectional.

to be considered beyond the health sector that serves as a baseline for decision making in various sectors. ${ }^{16}$ The effective utilisation of DHIS improves reliable and timely health information. This, in turn, will serve as vital for operational and strategic decision making that saves lives and enhances the quality of health. ${ }^{7-9}$

Currently, DHIS is used in more than 60 countries and most global initiatives are more interested in using DHIS for monitoring health performance. ${ }^{1} 1011$ Ethiopia has also produced its potentials for DHIS utilisation that deploy user-friendly DHIS versions to the whole region. The Federal Ministry of Health $(\mathrm{FMOH})$ is deploying and implementing DHIS to enhance decision making among public health facilities.

The Ethiopian FMOH has taken DHIS as an important and guiding programme management and policy development to extract and use data for decision making and taken as a national electronic health management information system. This is used to promote the four transformation agendas in the country's health sector transformation plan which is 'Information Revolution'. ${ }^{12-15}$ DHIS is 
one of the building blocks of a health system to improve access and quality of service delivery through evidencebased practice. ${ }^{16}$ The gathering and summarising of data through DHIS software provides effective health services that are supported by evidence. ${ }^{17}$

The issues of data quality and completeness are affected by many factors that need to be assessed at every health facility. A low level of data utilisation for decision making among health professionals can compound low motivations for data utilisation among the staff. The data generated are incomplete, inaccurate and erratic affecting managers' self-confidence to use data. ${ }^{18-20}$ The facilities/ organisations are expected to promote a culture of information use, increase their competence in conducting health information system (HIS) tasks that will help to improve the quality of health service. ${ }^{321}$ This will, in turn, helps increase confidence in carrying out HIS tasks for decision-making purposes. ${ }^{1620}$

As the study revealed, the prevalence of DHIS utilisation was $72.3 \%$ where the data are required for public decision making. ${ }^{19}$ In Kenya, $69.7 \%$ of data is used for decision making that helps the stakeholders to form policy and plan. ${ }^{22}$ As the study shows, ${ }^{23} 34 \%$ of respondents were using routine generated data for their decision-making purposes, while the study identified that level of data utilisation from DHIS was $60 \% .{ }^{24}$ Over the last 2 years alone, about $90 \%$ of the data were produced but it is found that there is an increasing gap between data acquisition and use for decision making. ${ }^{25}$

According to the Ethiopian FMOH, at the same time, this would be possible whenever there is an effective and efficient use of DHIS at each health facility. The ministry has said there is a need for stakeholders to work collaboratively to ensure its success. In Ethiopia, despite the health information revolution is one of the key components of health system strategies and plans, the quality and use of data are reported to be weak, particularly in the primary healthcare (PHC) facilities. ${ }^{16}$ The same is true for the area where this study was conducted.

Despite the high demand for quality data at PHC levels, evidence shows immense challenges in the utilisation of health-related data including Ethiopia. Inadequacy of human and financial resources, low management support, lack of supervision and leadership which are common to every health facility in sub-Saharan countries, has a significant impact on the utilisation of DHIS. ${ }^{20} 26$ Effective utilisation of data is required because reliable, accurate, and timely information is vital for the provision of quality health service. ${ }^{152728}$ However, the utilisation of the DHIS in the southwest of Ethiopia remains as a gap and has not been assessed yet. The result of this study is expected to help health facilities located in the southwest of Ethiopia and their administrative health office by increasing their culture of information use. Health facilities and researchers can use this study as a baseline and explore further study. Therefore, this study was conducted to assess the extent of DHIS utilisation and its determinant factors among health professionals working in the public health facilities of the southwest of Ethiopia. In addition, the study has assessed the extent of utilisation and determinant factors at different kinds of health facilities including health centres and hospitals. Even if there is a lot of studies throughout the world, there is no much study conducted in the southwest of Ethiopia related to the utilisation of DHIS. Generally, the study aimed to assess utilisation of DHIS and the factors that will hinder the extent of utilisation.

\section{METHODS}

\section{Study design and setting}

A quantitative cross-sectional study design was conducted among health facilities in Illu Aba Bora zone from January to February 2020. Illu Aba Bora zone is located in the southwest of Ethiopia at $600 \mathrm{~km}$ away from Addis Ababa and it is one of the zones of Ethiopia's Oromiya region. According to the 2007 Census conducted by the CSA, this zone has a total population of 1271609 , an increase of 50.12\% over the 1994 census, of which 636986 are men and 634623 women; with an area of 15135.33 $\mathrm{km}^{2}$ and the zone has a population density of 84.02. This study covered different types of health facilities including health centres, primary hospitals and referral hospitals. For this study, (41 health centres, 2 hospitals (one referral hospital and one primary hospital) were approached and studied.

\section{Study subjects}

Source population

The source population of this study was health professionals who handle data, generate data, use generated data for their decision making, and those who serve as the focal person within their department.

\section{Study population}

The study population of this study was all selected health professionals who handle data, generate data, use generated data for their decision making, and those who serve as the focal person within their department/facilities. These health professionals were collectively known as the performance monitoring team according to the Ethiopian healthcare system.

\section{Inclusion and exclusion criteria Inclusion criteria}

All selected health professionals who handle data, generate data, use generated data for their decision making, and those who serve as the focal person within their department/facilities were included in the study.

\section{Exclusion criteria}

Health professionals who have less than 6 month service were excluded from this study.

\section{Sample size determination and sampling procedure}

Each study participant was approached, and information was collected. Every health facility within the study areas 
was approached and reached. Sample size was determined based on study participants of every health facility. Hence the number of study participants is very little (260), all health facilities and all health professionals were included in this study instead of using probability sampling.

\section{Operational definition}

Utilisation of DHIS

The utilisation of DHIS was measured using four questions of the Likert scale. The outcome variable was not measured in the study areas yet and the data were skewed and kurtosis in addition to the Shapiro-Wilk test $(p=0.05)$. Based on this assumption, we were obligated to use median over mean. Health professionals who scored the median value and above the median value were categorised as having good utilisation of DHIS for decision making whereas those who scored less than median value were categorised as having poor utilisation.

\section{Data collection tools and procedures}

A self-administered English version questionnaire was used to collect data. A five-point Likert scale survey questionnaire was employed to measure particular variables. Ratings were made on one to five scale where; $1=$ strongly disagree, $2=$ disagree, $3=$ neutral, $4=$ agree and $5=$ strongly agree. In addition to this, yes or no questionnaire was also employed to assess the factors as shown in online supplemental appendix 1 .

\section{Data quality control}

Data were collected by trained data collectors. Training was given for both data collectors and supervisors on the objectives of the study. Before actual data collection, a pretest was conducted among $5 \%$ of samples at Buno Bedele general hospital and health centre in Bedele town. The correctness, consistency and quality of the questionnaire were checked and seen in detail based on the pretest finding. The validity of the questionnaire was determined based on the view of experts and the reliability of variables was obtained by calculating the value of Cronbach's alpha $(\alpha=0.82)$.

\section{Data processing and analysing}

The data entry and analysis were done by SPSS V.20 and completeness was seen daily during data collection. Data cleaning was performed to ensure accuracy, consistency and missing values of variables by cross-checking the data found in SPSS. After checking, if something went wrong we have checked it in the original questionnaires. Any error that was identified during data entry was corrected after revision of the originally completed questionnaire. To explain the study population in relation to the relevant variables, descriptive statistics were used. Associations between dependent and independent variables were checked and their strength was presented using ORs and 95\% CIs. Both bivariate and multivariable logistic regression were used to assess the association between outcome and explanatory variables. A $\mathrm{p}<0.05$ was considered statistically significant in this study for multivariable.
Table 1 Sociodemographic characteristics of study participants at health facilities of llu Aba Bora Zone, 2020 $(\mathrm{n}=260)$

\begin{tabular}{lll}
\hline Variables & Category & Frequency (\%) \\
\hline Age & $\leq 30$ years & $184(70.8)$ \\
& $>30$ years & $76(29.2)$ \\
Sex & Male & $145(55.8)$ \\
& Female & $115(44.2)$ \\
Facility type & Hospitals & $28(10.8)$ \\
& Health centre & $232(89.2)$ \\
Experience & $\leq 3$ years & $60(23.1)$ \\
& $>4$ years & $200(76.9)$ \\
Position & Head position & $100(38.3)$ \\
& Expert position & $160(61.7)$ \\
\hline
\end{tabular}

The fitness of the model was checked by using Hosmer and Lemeshow test. Depending on bivariate logistic regression analysis, variables that were significant at $\mathrm{p} \leq$ 0.2 were considered candidates for the final model and 11 variables were entered into the multivariable analysis. Of this, five variables were significantly associated with the utilisation of DHIS for decision at $\mathrm{p}<0.05$.

Patient and public involvement

No patient involved.

\section{RESULT}

\section{Sociodemographic characteristics of study participants}

Out of the total sample, 260 study participants were involved in this study making the response rate $97 \%$. Health centres covered the majority of healthcare professionals $234(88.6 \%)$ and more than half the participants were male $145(55.8 \%)$. Respondents with the age of thirty and less than thirty years held about $70.5 \%$. Considering the study participants' position at their respective facility, 101 (38.3\%) of the study participants were serving on head positions at the facilities. Majority of the respondents $203(76.9 \%)$ had more than 4 years of working experience (table 1).

\section{The utilisation of DHIS for decision making}

The study identified that more than half of the respondents 149 (57.3\%) had good utilisation of DHIS for clinical decision making (95\% CI 50 to 64.2) (figure 1).

\section{Utilisation of DHIS by their socialdemographic factors}

Among a total of 145 male respondents, 80 (55.8\%) had good utilisation of DHIS whereas $69(60.0 \%)$ of female respondents had good utilisation. Respondents of age 30 and less than 30 had good utilisation of DHIS for their decision-making purpose. As for the facility types, respondents from hospitals had more good utilisation of DHIS $(60.7 \%)$ when compared with respondents from health centres (56.9\%). The study participants who were 


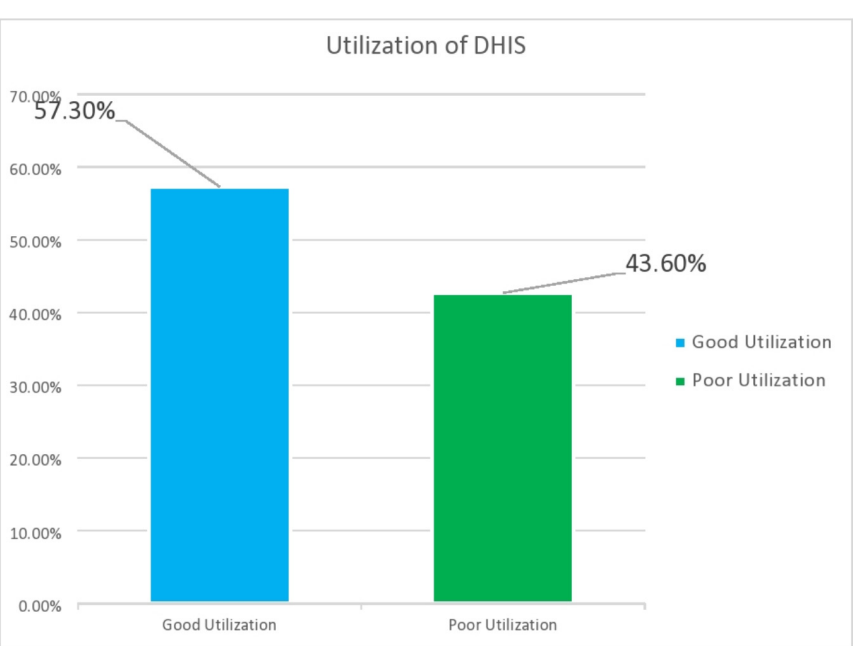

Figure 1 Utilisationof district health information system, good utilisation, poor utilisation

serving as the expert position had more good utilisation of DHIS (78 (49.1\%)) as compared with those serving as head position in their facilities. Having long experiences favours good utilisation of DHIS, as the study revealed that respondents with greater than 4 years had more good utilisation of DHIS (115 (57.5\%)) when compared with respondents with three and less than 3 years' experience $(34(56.7 \%))$ (table 2).

\section{Factors associated with utilisation of DHIS for decision making}

All variables with $p$ value $\leq 0.2$ in bivariate analysis were entered into the multivariable logistic regression to identify the association between dependent variable and independent variables. Five variables were significantly associated with utilisation of DHIS for decision making at $p \leq 0.05$ from the total 11 variables. Skills related to use DHIS, motivations to use DHIS, training, feedback and supervision supports were the factors associated with

Table 2 Utilisation of district health information system (DHIS data) by their sociodemographic characteristics $(n=260)$

\begin{tabular}{llrr}
\hline & & \multicolumn{2}{c}{ Utilisation of DHIS (n=260) } \\
\cline { 3 - 4 } Variables & \multicolumn{1}{l}{$\begin{array}{l}\text { Good } \\
\text { utilisation } \\
\text { frequency (\%) }\end{array}$} & $\begin{array}{l}\text { Poor } \\
\text { utilisation } \\
\text { frequency (\%) }\end{array}$ \\
\hline Sex & Female & $69(60.0)$ & $46(40.0)$ \\
Ages & Male & $80(55.8)$ & $65(44.2)$ \\
Type of & $\leq 30$ years & $109(59.2)$ & $75(40.8)$ \\
facilities & Hospital & $17(60.7)$ & $11(39.3)$ \\
Position at & Health centre & $132(56.9)$ & $100(43.1)$ \\
facility & Expert position & $78(49.1)$ & $82(50.9)$ \\
Experiences & Head position & $40(40.6)$ & $60(59.4)$ \\
& $\leq 3$ years & $34(56.7)$ & $26(43.3)$ \\
& $>4$ years & $115(57.5)$ & $85(42.5)$ \\
\hline
\end{tabular}

health professionals' utilisation of DHIS for decision making. The skills related to DHIS could determine the health professionals' utilisation of DHIS for their decision making. Those health professionals who had sufficient skills related to DHIS were more likely to have good utilisation of DHIS for decision making when compared with health professionals with insufficient skills (AOR 3.83, 95\% CI 1.92 to 7.64). The manner of feedback among health professionals within their facilities also matters the status of utilisation of DHIS for decision making. Health professionals who had received feedback were about 2.97 times (AOR 2.97, 95\% CI 1.53 to 5.77) more likely to be good at utilisation of DHIS for decision making when compared with those who had not received feedback.

Within their respective facilities, health professionals need to have supervision supports that will promote them to use DHIS efficiently and effectively. Thus, health professionals with supervision and managerial support regarding the use of DHIS were about 3.06 times (AOR $3.06,95 \%$ CI 1.56 to 6.01 ) more likely to be good at utilisation of DHIS for decision making than those who have no supervision.

The odds of study participants who have sufficient training related to DHIS were about 3.90 times (AOR $3.90,95 \%$ CI 1.95 to 7.79 ) more likely to be good at utilisation of DHIS than those who have no sufficient training in DHIS. Similarly, the odds of health professionals having high motivation to DHIS were 3.93 times (AOR 3.93, 95\% CI 1.99 to 7.76) more likely to be good at utilisation of DHIS as compared with health professionals who have low motivation (table 3).

\section{DISCUSSION}

The study found that the overall utilisation of DHIS was 149 $(57.3 \%)$, which was similar to the study conducted in the East Wollega zone, Oromia regional state, Ethiopia. ${ }^{16}$ The result of this study was lower than the study conducted in Ghana. ${ }^{4}$ The possible explanations for this variation could be due to the extent of the study area and scope of the study. The study in Ghana included district, community and other health offices, but the present study focused only on the public health facilities. In addition to this, the deployment of DHIS in Ghana has counted longer time than that of Ethiopia. This result was also lower than the study conducted in Kenya. ${ }^{22}$ The difference could be due to sample size and study design. However, the result of this study was greater than the study ${ }^{23}$ which indicated that the utilisation of routine health information was $34 \%$. This might be because the government of Ethiopia has given special attention to the utilisation of HIS for decision making and the internal commitment of healthcare providers to use data. To the extents, this study was too far from the study conducted in Botswana which reported that only $11 \%$ were used for decision-making purposes. ${ }^{29}$ This might be due to differences in study periods because technology runs fast, availability of trained human power, infrastructures, government's emphasis and accountability for information use. 
Table 3 Univariate and multivariable logistic regression analysis for factors associated with utilisation of DHIS data among health professionals at health facility in Ilu Aba Bora zone, Oromia region, Ethiopia $2020(n=260)$

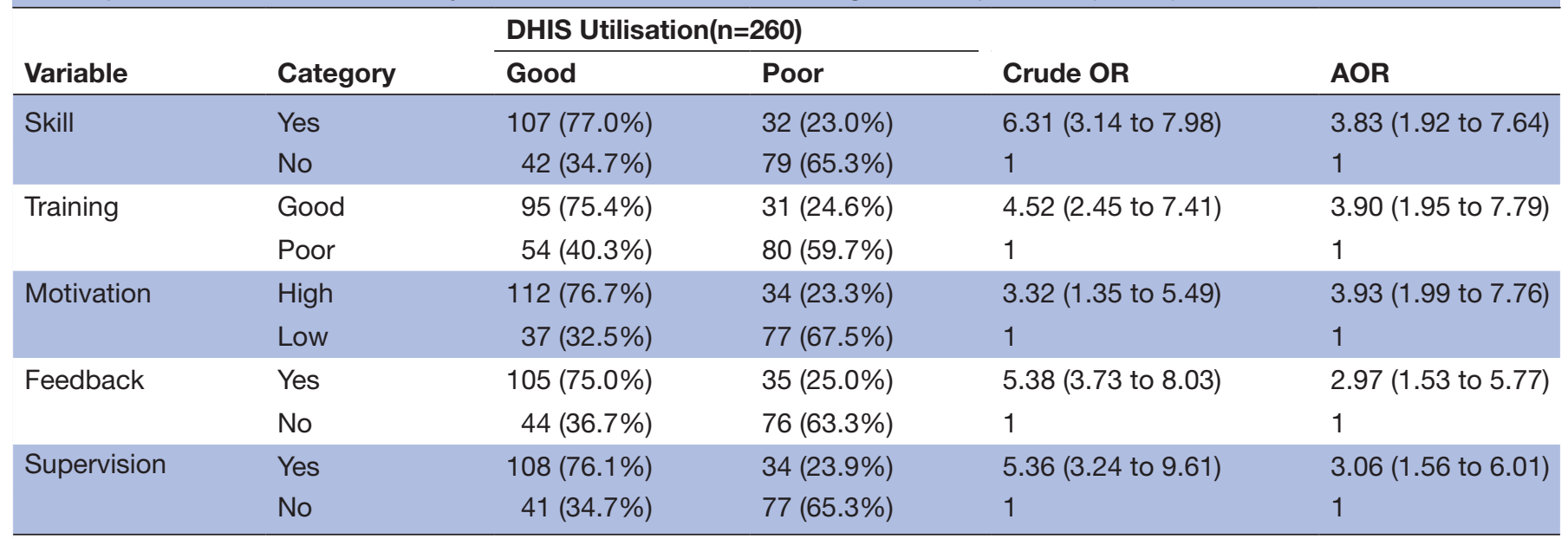

DHIS, district health information system.

Additionally, an increased awareness towards the HIS over a period of time and sample size could also matter the variations. The study conducted in Liberia also perceived that there is poor utilisation of routine HIS which was lower than the result of this study. ${ }^{30}$ The possible difference might be due to the nature of study participants. The participants in the former study were those who participate only in policymaking and participated in decision making within health.

The study conducted in Jimma, Ethiopia was lower than the result of this study, which revealed that the rate of routine HIS utilisation was $32.9 \% .^{27}$ In the same way, the studies conducted in Eastern Ethiopia, ${ }^{31}$ Southern Ethiopia, ${ }^{32}$ and Western Amhara Ethiopia ${ }^{33}$ were lower than the result of this study. This difference might be happened because of the attention given by both government and health professionals on the utilisation of DHIS.

According to this study, the odds of health professionals who had sufficient skills were 3.83 times more likely to have good utilisation of DHIS for decision making when compared with respondents who had insufficient skills (AOR 3.83, 95\% CI 1.92 to 7.64). This result was consistent with the study conducted in Kenya. ${ }^{34}$ It was also supported by another study, ${ }^{35}$ which showed that skills related to data interpretations and analysis were directly associated with utilisation of routine HIS. The study conducted in Ethiopia also revealed similar findings. ${ }^{36}$ Another study conducted in the western Amhara region of Ethiopia revealed that having good skills was positively associated with the utilisation of DHIS ${ }^{33}$ and Columbia. ${ }^{37}$ Many other studies also revealed that utilisation of health information could be determined based on the sufficiency of health professionals' skills. ${ }^{6} 16273233$

The good utilisation of DHIS was also associated with being trained (AOR 3.90, 95\% CI 1.95 to 7.79). This result was supported by the study conducted in Ethiopia which showed being trained about health information was positively correlated with utilisation of HIS. ${ }^{33}$ Other studies conducted in Ethiopia and Ghana stated that being trained was positively associated with utilisation of DHIS. $^{638}$ The possible reason might be because usage and interpretation of data captured from training would enhance DHIS decision making.

The present study also revealed that high level of motivation was associated with utilisation of DHIS (AOR 3.93, 95\% CI 1.99 to 7.76 ). This result was consistent with the studies conducted in Ghana ${ }^{39}$ and East Wollega zone, Oromia region of Ethiopia. ${ }^{16}$ This might be due to the fact that health professionals who received rewards for their good works and who are motivated to use data had always good utilisation of DHIS for decision making.

The odds of DHIS utilisation among health professionals who were given regular feedback on DHIS utilisation were greater than those who were not given feedback (AOR 2.97, 95\% CI 1.53 to 5.77). This result was supported by the studies conducted in Jimma, Oromia Ethiopia. ${ }^{6239}$ The possible explanation could be because the culture of receiving and providing feedback to either of coworkers will facilitate the utilisation of routine HIS for decision making. ${ }^{31}$ The odds of having supportive supervision among health professionals were greater than who do not have supportive supervision (AOR 3.06, 95\% CI 1.56 to 6.01 ). This finding was supported by the studies conducted in Kenya, Ghana and Ester Ethiopia. 20313940

\section{CONCLUSION}

In general, more than half of health professionals showed a good level of DHIS utilisation 149 (57.3\%). Skills, training, supportive supervision, feedback and motivation to use DHIS were the most determinant factors for DHIS utilisation. The study has found significant factors that affect the utilisation of DHIS for their decision making. An attempt to provide training, supportive supervision, skills related to DHIS use, promotion of motivation and feedback will help to improve and achieve the expected utilisation of DHIS for decision making. 


\section{Limitation of the study}

Though this study was aimed to assess utilisation of DHIS and its determinant factors, this was not to mean that it was immune to certain constraints. These data were collected within a short period of time and time limitations might have affected the overall utilisation of DHIS. The study was restricted to only health professionals serving as head/focal persons. This could also affect the generalisability of the result. In addition, it is difficult to know about the precedence of the problem in detail because the study was cross-sectional. The small sample size used could be another limitation of this study.

\section{Recommendation}

If policy-makers, actors and other expected researchers do not focus on the utilisation of DHIS, the goal and objectives of health service would not be achieved as expected as possible. Therefore, supervision and provision of feedback for their respective employees in the health facilities are encouraged.

Contributors All authors made substantial contributions to the conception and design of the study. For instance, SGK and MHA contributed to the conceptualisation and designing, acquisition of data, interpretation of data and writing up of the first draft of the manuscript. HSN and RDB mostly produced their potentials on data collection and analysis. GRD, MHA and SGK contributed to the second draft of the manuscript. HSN and RDB performed data interpretation and writing of the revised manuscript.

Funding The authors have not declared a specific grant for this research from any funding agency in the public, commercial or not-for-profit sectors.

Competing interests None declared.

Patient consent for publication Not required.

Provenance and peer review Not commissioned; externally peer reviewed.

Data availability statement Data are available on reasonable request.

Supplemental material This content has been supplied by the author(s). It has not been vetted by BMJ Publishing Group Limited (BMJ) and may not have been peer-reviewed. Any opinions or recommendations discussed are solely those of the author(s) and are not endorsed by BMJ. BMJ disclaims all liability and responsibility arising from any reliance placed on the content. Where the content includes any translated material, BMJ does not warrant the accuracy and reliability of the translations (including but not limited to local regulations, clinical guidelines, terminology, drug names and drug dosages), and is not responsible for any error and/or omissions arising from translation and adaptation or otherwise.

Open access This is an open access article distributed in accordance with the Creative Commons Attribution Non Commercial (CC BY-NC 4.0) license, which permits others to distribute, remix, adapt, build upon this work non-commercially, and license their derivative works on different terms, provided the original work is properly cited, appropriate credit is given, any changes made indicated, and the use is non-commercial. See: http://creativecommons.org/licenses/by-nc/4.0/.

ORCID iDs

Shuma Gosha Kanfe http://orcid.org/0000-0001-9118-296X

Habtamu Setegn Ngusie http://orcid.org/0000-0002-5477-9748

\section{REFERENCES}

1 Farnham A, Utzinger J, Winkler MS. Using district health information to monitor sustainable development. Bull World Health Organ 2015.

2 WHO. Support tool to assess health information systems and develop and strengthen health information strategies, 2015. Available: https://www.euro.who.int/_data/assets/pdf_file/0011/ 278741/Support-tool-assess-HIS-en.pdf

3 Garrib A, Stoops N, McKenzie A. An evaluation of the district health information system in rural South Africa. S Afr Med J 2008;98:549-52.
4 Odei-Lartey EO, Prah RKD, Anane EA, et al. Utilization of the National cluster of district health information system for health service decision-making at the district, sub-district and community levels in selected districts of the Brong Ahafo region in Ghana. BMC Health Serv Res 2020;20:1-15.

5 Kawale P. Determinants of use of health information in Nathenje health area of Lilongwe district. University of Malawi, 2011.

6 Wude $\mathrm{H}$, Woldie M, Melese D, et al. Utilization of routine health information and associated factors among health workers in Hadiya zone, southern Ethiopia. PLoS One 2020;15:e0233092.

7 Amaniampong RO, Agyei-Baffour P, Daniel B. Knowledge of health information for healthcare decision making: a cross sectional study of health staff in Kumasi Metropolis, Ghana. $J$ Health Med Nurs 2017;40:30-7.

8 Belay H. Prism tools application, 2013.

9 Nicol E, Bradshaw D, Phillips T. Human factors affecting the quality of routinely collected data in South Africa. MEDINFO. los Press, 2013: 788-92.

10 Dehnavieh R, Haghdoost A, Khosravi A, et al. The district health information system (DHIS2): a literature review and meta-synthesis of its strengths and operational challenges based on the experiences of 11 countries. Health Inf Manag 2019;48:62-75.

11 Farnham A, Utzinger J, Kulinkina AV, et al. Using district health information to monitor sustainable development. Bull World Health Organ 2020;98:69-71.

12 Avan BI, Berhanu D, Umar N, et al. District decision-making for health in low-income settings: a feasibility study of a data-informed platform for health in India, Nigeria and Ethiopia. Health Policy Plan 2016;31(Suppl 2):ii3-11.

13 Hoyiso D, Arega A, Markos T. Evidence based nursing practice and associated factors among nurses working in Jimma zone public hospitals, Southwest Ethiopia. Int J Nurs Midwifery 2018;10:47-53.

14 Gebre-Mariam M. Governance lessons from an interorganizational health information system implementation in Ethiopia. E J Info Sys Dev Countries 2018;84:e12045.

$15 \mathrm{FMoH}$. Ethiopia's experience on strengthening community health information systems, data quality and data use 2016.

16 Kebede M, Adeba E, Chego M. Evaluation of quality and use of health management information system in primary health care units of East Wollega zone, Oromia regional state, Ethiopia. BMC Med Inform Decis Mak 2020;20:1-12.

17 Managing data wi th dhis2 : improving health commodities reporting and decision making in Kenya 2020.

18 Kuyo RO, Muiruri L, Njuguna S. Organizational factors influencing the adoption of the district health information system 2 in Uasin Gishu County, Kenya. International Journal of Medical Research \& Health Sciences 2018;7:48-57.

19 Kirimi NS. Factors influencing performance of routine health information system: the case of Garissa Subcounty. Kenya: University of Nairobi, 2017.

20 Ndegwa CW. Assessment of data quality and information use of the community health information system: a case study of Karurumo community health unit-Embu County. Kenya: University of Nairobi, 2015.

21 Raeisi A, Saghaeiannejad S, Karimi S, et al. District health information system assessment: a case study in Iran. Acta Informatica Medica 2013;21:30-5.

22 Njoka PM. Factors influencing utilization of routine health data in evidence based decision making in HIV/AIDS services by public health facilities in Nakuru County: University of Nairobi. Imp J Interdiscip Res 2015;58:538-45.

23 Determinants of utilization of routine data 2013.

24 Gathua PW. Assessment of data use of the district health information system (dhis2): a case study of Nairobi County. University of Nairobi, 2016.

25 Chen Y, Elenee Argentinis JD, Weber G. Ibm Watson: how cognitive computing can be applied to big data challenges in life sciences research. Clin Ther 2016;38:688-701.

26 Nutley T, Reynolds $\mathrm{H}$. Improving the use of health data for health system strengthening. Glob Health Action 2013;6:20001-11. 1):1.

27 Abajebel S, Jira C, Beyene W. Utilization of health information system at district level in jimma zone oromia regional state, South West Ethiopia. Ethiop J Health Sci 2011;21:65-76.

28 Shiferaw AM, Zegeye DT, Assefa S, et al. Routine health information system utilization and factors associated thereof among health workers at government health institutions in East Gojjam zone, Northwest Ethiopia. BMC Med Inform Decis Mak 2017;17:1-9.

29 Seitio-Kgokgwe O, Mashalla Y, Seloilwe E. Utilization of the district health information software (DHIS) in Botswana: from paper to electronic based system 2016;3:1-10. 
30 Performance of routine health information system management in Liberia 2014:1-41.

31 Teklegiorgis K, Tadesse K, Mirutse G. Factors associated with low level of health information utilization in resources limited setting, eastern Ethiopia. IJIIS 2014;3:69-75.

32 Zegeye AH, Kara NM, Bachore BB. Utilization of community health information system and associated factors in health posts of Hadiya zone, southern Ethiopia 2020;63:13-22.

33 Asemahagn MA. Determinants of routine health information utilization at primary healthcare facilities in Western Amhara, Ethiopia. Cogent Medicine 2017;4:1387971.

34 Otieno MO, Muiruri L, Kawila C. Organizational determinants of health information utilization in making decision among healthcare managers in Mombasa County, Kenya 2020.

35 Hotchkiss DR, Aqil A, Lippeveld T, et al. Evaluation of the performance of routine information system management (PriSM) framework: evidence from Uganda. BMC Health Serv Res 2010;10:1-17.
36 Seid MA, Bayou NB, Ayele FY, et al. Utilization of routine health information from health management information system and associated factors among health workers at health centers in Oromia special zone, Ethiopia: a multilevel analysis. Risk Manag Healthc Policy 2021;14:1189-98.

37 Tamime A. No TitleE $\Lambda$ ENH. A $\gamma \alpha \eta$. 201;8:55.

38 Alawiye R. Use of aggregate data for health decision making at district level: case study of GA West Municipality of the greater Accra region. University of Ghana, 2019.

39 Effah FD. Commitment among senior managers to the use of district health information management system 2 data for decision making in maternal and neonatal health in greater Accra region. University of Ghana, 2019.

40 Okyere Boadu R, Adzakpah G, Agyei-Baffour P. The role of quality improvement process in improving the culture of information among health staff in Ghana. Adv Public Health 2019;2019:1-9. 